\title{
Blockchain and the Internet of Things: Challenges in Monitoring System for Covid-19
}

\author{
SHOAIB UL HASSAN ${ }^{1}$, JINGXIA CHEN ${ }^{2}$, ALI AKBAR ${ }^{3}$, \\ ZAKI RAMZAN ${ }^{4}$, MUHAMMAD AHSAN ${ }^{5}$, MUHAMMAD \\ ASIM HAFEEZ 6 \\ 1,2,3,4,5 Department of Electronic Information and Artificial Intelligence, \\ Shaanxi University of Science and Technology \\ Xi'an, China \\ ${ }^{6}$ Department of Gender Studies \\ Quaid-i-Azam University Islamabad, Pakistan \\ DOI: 10.29322/IJSRP.11.07.2021.p11597 \\ http://dx.doi.org/10.29322/IJSRP.11.07.2021.p11597
}

\begin{abstract}
The COVID-19 (Corona Virus) is an intensely rising virus that has intentioned countries to utilize technologies to identify Covid disease. Several countries are working incredibly to battle against COVID-19. Numerous countries have been utilizing a range of gadgets to contest the pandemic, looking for data about growth, perceiving as well as the emancipating the personal data of the inhabitants. In the present situation of COVID-19 (Corona Virus) legislative institutes all around the world make an effort to build a monitoring system that notifies about declared cases throughout the world. Several Researcher suggested IoT and Blockchain-based applications for this reason. The significant thought behind a IoT and Blockchain-based system for monitoring is quality services, information handling and prevent from Single Point of Failure. Even now , it raises various issues concerning to Security Issues, Legal Disputes, Privacy Requirements, Latency, Throughput, Scalability, Intense Traffic and Block Size. This article aims to discuss these IoT and Blockchain-based applications Challenges.
\end{abstract}

\section{Keywords-- COVID-19, IoT, Blockchain}

\section{INTRODUCTION}

The Whole World are fighting with another disease called Corona Virus. It is first distinguished in the Wuhan City of China in end of the Dec 2019. In Mainland China it began a lot of deaths [1]. After 10 Mar 2020, The World Health Organization (WHO) uttered corona virus an overall pandemic. At the time of writing article, more than 172M cases and more then $3.69 \mathrm{M}$ deaths have been recounted for in the additional of 189 nations and locales.

Technological innovations are the perilous assets of the modern world that may assist us defeating the difficulties given by corona virus. New innovations-like Internet of Things (IoT), Blockchain, Machine Learning (ML), Big Data, Artificial Intelligence (AI), robotics, 3D prints, 5G interchanges, nanotechnology, cloud \& edge computingcould be utilized to build intellectual disaster managing policies for the corona virus. Eminently, Block-chain has been distinguished by European Parliamentary Research Service (EPRS) as most important key developments to battle against corona virus [2]. The blockchain offers a distributed computational facility architecture that eliminates the vast majority of the restrictions related with the integrated computing network. Blockchain is an variety of computing hubs that are linked in a distributed peer-topeer (P2P) method and commonly check transactions implemented inside the network [20]. In the Blockchain, every block cryptographically closes a bunch of transaction and is connected with prior block to construct a hash based (cryptographically ) group of blocks.

Blockchain is a decentralized database to keep the history of transaction along with timestamps[3,20]. Blockchain begin through a primary block known as a genius block. Private and public keys are utilized to access the network as well as for transaction. The node creates a transaction approved with its private key and communicated through the network. Different nodes on the network approve this transaction and rejected if it is invalid[4]. 
In the present situation of COVID-19 legislative institution all over the world make an effort to develop a monitoring system that clarifies about declared cases.

Numerous research proposed a Blockchain-based system. Meanwhile Blockchain is a distributed ledger of blocks that hold all transaction record transparently. To examine the situation around hubs and nodes requires to send query messages to the network nodes by connecting with the Blockchain network through its public key and signed the message with its private key[3]. Once receiving the message hub initially confirms the authentication and then look for data that it holding and notify the requesting hub/nodes. The message of requesting hub is communicated all over the great no of connected hubs. This distributed system has numerous befits however has a few issues related to data quality and data handling.

\section{Smart Apps to Detect the coronavirus}

The Covid applications are cell phone programs that utilized Electronic connection tracking for countering to the Covid pandemic. Different versatile applications are creating in certain countries, through supported government financing. Numerous different methodologies are proposed for creating contact detecting apps. Like the COVID-19 virus outbreak panics, researchers are presently rushing all-around to make contact finding gadgets, projects, and apps for perceiving and alerting each and every individual who falls into contact with infected individuals. A few apps are created by various countries. Some of them are labeled as

\section{A. Covid Watch}

This application was made through an association with Stanford University, (United State) the genuinely courageous people to help themselves and the areas while surrendering secrecy. This uses Bluetooth signals to distinguish people at whatever point they are near each other, or subtly cautions people when they're in contact with infected people. The particular part of the application is that each accomplice, similar to the specialists, can't monitor who has been accounted for in which. With private data preserving, community Bluetooth touch tracking, this was one of the main applications to introduce an open source specification [5].

\section{B. Trace Together}

It is intended to permit community driven touch tracking to help progressing local activity to handle the Corona virus pandemic. This application was introduced by the Government of Singapore that uses the modified Blue trace standard to empower tracking digitally[6]. It was
Published on 20 March 2020, by Public IT Department. It has been retrieved by $17 \%$ of the absolute population since the starting of the application, which implies in excess of 2,800,000 clients.

\section{Aarogya Setu}

This application records situating data that require an ordinary connection with the Mobile gadget, which is disturbing from a security $\&$ privacy point of view. As well as to work with lawful consistence rules, and so forth [7].

\section{Let's Beat COVID}

It is launched to offer peoples to perform a short conversation in regards to fitness involving Corona virus danger in efforts to save more life of individuals [8]. This was distributed by Med Shr, a clinical app used by in excess of 1,000,000 doctors. Residents are mentioned to review out a short online poll regarding themselves, but at the same time they're needed to link in their family members' details.

\section{E. Other Apps}

Many applications are developed, for example, NHS, Covid-19 Tracker, Corona Data, COVID Symptom Tracker[9], and so forth and a lot of portable apps are under development stages

\section{Analysis on currently working apps of COVID-19}

In this article [4] author presented, a descriptive analysis of all applications associated with Corona virus. Author searched Google Play Store and IOS App Store for Corona Virus related apps. After collecting the apps information, author categories information intofollowing classes
a) News Report
b) Help Desk
c) Monitoring
d) Self-Diagnosis
e) Contact tracing
f) No of close cases
g) Overall information
h) Record of symptoms and treatment
i) Contact with health care professionals

After inquiry author determined that the applications' most common objectives are providing data on the no of recovered, and infected. These applications gave the recorded information instead of Realtime assessment Johns Hopkins security and health center announced a national wide methodology to save the lives, decline in Corona virus load on the medical services system, and 
fearlessly make development toward getting back to the working environment and educational institutes [10]. The authors suggested that the US basically utilize the robust and expansive framework to perceive all Corona virus cases and distinguishes all associations of each detected case. As per the evaluation, if 1 influenced patient spreads the infection to 3 other patience, that first positive case can go into 58,000 cases in just about 10 circles of infections. Corona virus is recently disseminated over social orders through the US. so, this methodology will be unbearable to accomplish for Corona virus without a novel nationwide inventiveness that assemble a colossal development of quick diagnostic assessments with the acknowledgment of novel apparatuses for ID of cases and association tracing in singular state. To prevail in the management of Corona virus epidemics from right now to onward, societies in the US need:

- Quick diagnostic evaluations for all cases

- The ability to distinguish all cooperation's of each recognized case.

To accomplish this target needs actions to made by administrative and other association to stand up with the abilities quickly. Numerous Countries have been taking part in to be capable for contact tracing. In South Korea, the organization has maintained a local area databank of recognized patients, containing information on their present travel routes, age, profession and sex [11]. In Israel, guideline was supported to allow the organization to follow public cell phone information who suspected with disease [12]. In Taiwan, healthcare organizations were given the privilege to check the patients' traveling history [13], and foundations reserve the option to get to the telephone site statistics for anyone under quarantine [14]. Additionally, the Government of Singapore delivered a versatile application, Trace-Together is an app launched to help healthiness reps trace the experiences after the ID of infected peoples. Yet, there are huge confidentiality inferences from these applications. Though Singapore's Trace Together app has different security issues [14].

Author [15] proposed a tracking framework that makes use of Block-chain based Smart Contracts of Ethereum and oracles for tracing information related with the figure of new cases, recovered cases and death which is gotten from solid sources. Figures identified with
a) No of Hospital Bed Occupied
b) Patients hospitalized
c) Positive and Negative tests
d) Deaths
e)Ventilator Shortfalls

This information helps the administrators trace the Corona virus circumstance in real time. But, these caries a fundamental issue as decisions grounded on such realities are frequently imperfect and deficient. Moreover, tracing legitimate information is imperative to investigate the pandemic circumstance [16,17]. Data Accessible online potentially imperfect as it is defenseless for the data operations. Authors claims that Block-chain innovation can change the strategy for tracing Corona virus cases. They concentrating on the benefits of applying a Block-chain goal over a customary centralized solution. But there are a few issues in execution of Blockchain based solution for monitoring Corona virus.

\section{A. Tracking}

The tracking [18] of Network information traffic flows turns immense as the no of transactions rises each day. Each and every hub on the blockchain stores history of every confirmed transactions, and subsequently this ends up being an issue via limitation on the block size and time frame utilized for the formation of a new block. Presently, the platforms of blockchain can deal with restricted or a couple of transactions each second. This transforms into an issue of blockchain for use in real time IoT applications as billions of transactions are necessary to be processed in a real time environment.

\section{B. Scalability}

Proof-of-work (POW) required a great deal of computational power. Nonetheless, IoT gadgets are restricted in resources. As the IoT network comprises of countless necessities so this blockchain network inadequately scaled because of the computational power needed for POW $[19,20]$.

\section{Latency}

The block size is restricted, this turns into the beginning for roughly a couple of transactions to be deferred. Therefore, the issue of latency features that from the perspective of latency, tracing by utilizing a block-chain platform isn't appropriate. This issue makes it problematic to comprehend the block-chain based trading platform. Moreover, blockchain required huge bandwidth and a lot of computational power. In this way, blockchains are not completely appropriate for restricted resources IoT gadgets proposed for smart cities [21]. All things considered, the blockchain used to determine the safety 
and secrecy issues of clinical related information. An improved blockchain based strategy was anticipated, which is fitting for IoT gadgets rely upon distributed network for additional security and privacy [22]. In [23], the author assemble the Internet of Medical Devices (IOMT) applications and blockchain in clinical for analysis of patients' information and study for good therapy.

\section{Discussion}

Corona virus is a major group of infections thatinfluence people from a basic virus to additional genuine conditions. The epic Covid is presently another illness unidentified among individuals before. Covid is profoundly pathogenic which implies that it very well may be spread among people and animals [24]. Sore throat, fever, severe symptoms, shortness of breath, and cough are similar example of diseases. With more dire conditions, kidney disease, pneumonia, outrageous respiratory misery condition as well as deaths may result from infections. The situation demonstrates that now the Covid might have been moved by the symptomless transporter. Typical rules for forestalling transmission of the infection include every day washing face, hand, and noses safeguarding while sneeze or cough, cautiously getting ready dinners of meat items. Versatile based testing was done to distinguish malaria, HIV, tuberculosis, and different poisons in the meals. Exploration has additionally been continuous to utilize mobile to distinguish corona virus however there are various concerns in regards to the effortlessness and adequacy that utilization advancements toward this path. Blockchain innovations currently can possibly change medical services frameworks. This offers a motivator to advance fitting business sector approaches in the medical sector, however. Reliable sharing of patient information may lead not exclusively to things turning out to be led suitably, and furthermore to the utilization of new methodologies. Effectively giving individuals the authority over their wellbeing records will be a portion of the key significant impacts. Blockchain innovation will hypothetically require authorization and access to clinical information to be overseen by patients at whatever point they see it commendable.

\section{Conclusion}

The corona virus pandemic has influenced numerous areas of life, Like politics, healthcare, education, economics and finance. Block-chain can perform a crucial part in the administration of the corona virus world. In the current circumstance of the corona virus all through the need to foster an monitoring framework that advises about confirmed cases around. Numerous researchers proposed a Block-chain based apps for this reason. However, a assortment of difficulties, for example, throughput, tracing, traffic, security, latency, scalability, block size, privacy and resource consumption issues should be settled before the blockchain can be completely used for all these reasons. This article presents the various works on the corona virus monitoring system and the issues of Blockchain that needs to be resolved.

\section{REFERENCES}

[1] Budaev, Andrey. "The coronavirus pandemic in the Latin America: factor of the rollback to the past or a drive for development?." Latin America 9 (2020): 6-23.

[2] Kalla, Anshuman, Tharaka Hewa, Raaj Anand Mishra, Mika Ylianttila, and Madhusanka Liyanage. "The role of blockchain to fight against COVID-19." IEEE Engineering Management Review 48, no. 3 (2020): 85-96.

[3] Sultan, Abid, Muhammad Azhar Mushtaq, and Muhammad Abubakar. "IOT security issues via blockchain: a review paper." In Proceedings of the 2019 International Conference on Blockchain Technology, pp. 60-65. 2019.

[4] Collado-Borrell, Roberto, Vicente Escudero-Vilaplana, Cristina Villanueva-Bueno, Ana Herranz-Alonso, and Maria Sanjurjo-Saez. "Features and functionalities of smartphone apps related to COVID-19: systematic search in app stores and content analysis." Journal of medical Internet research 22, no. 8 (2020): e20334.

[5] Alam, Tanweer. "Internet of things and blockchain-based framework for coronavirus (covid-19) disease." Available at SSRN 3660503 (2020).

[6] TraceTogether, Team. "How does TraceTogether work."

[7] App, Aarogya Setu Mobile. "MyGov. in.(2020)."

[8] Udugama, Buddhisha, Pranav Kadhiresan, Hannah N. Kozlowski, Ayden Malekjahani, Matthew Osborne, Vanessa YC Li, Hongmin Chen, Samira Mubareka, Jonathan B. Gubbay, and Warren CW Chan. "Diagnosing COVID-19: the disease and tools for detection." ACS nano 14, no. 4 (2020): 3822-3835.

[9] Rodriguez-Morales, Alfonso J., D. Katterine BonillaAldana, Ruchi Tiwari, Ranjit Sah, Ali A. Rabaan, and Kuldeep Dhama. "COVID-19, an emerging coronavirus infection: current scenario and recent developments-an overview." J Pure Appl Microbiol 14, no. 1 (2020): 5-12.

[10] Watson, C., A. Cicero, J. Blumenstock, and M. Fraser. "A national plan to enable comprehensive COVID-19 case finding and contact tracing in the US Baltimore." MD: Johns Hospkins University (2020).

[11] Kim, Min Joo, and Simon Denyer. "A 'travel log'of the times in South Korea: Mapping the movements of coronavirus carriers." The Washington Post (2020).

[12] Tidy, Joe. "Coronavirus: Israel enables emergency spy powers." BBC News, March 2020 (2020).

[13] Wang, C. Jason, Chun Y. Ng, and Robert H. Brook. "Response to COVID-19 in Taiwan: big data analytics, new 
technology, and proactive testing." Jama 323, no. 14 (2020): 1341-1342.

[14] Lee, Yimou. "Taiwan's new'electronic fence'for quarantines leads wave of virus monitoring." Reuters Technology News, March (2020).

[15] Cho, Hyunghoon, Daphne Ippolito, and Yun William Yu. "Contact tracing mobile apps for COVID-19: Privacy considerations and related trade-offs." arXiv preprint arXiv:2003.11511 (2020).

[16] Marbouh, Dounia, Tayaba Abbasi, Fatema Maasmi, Ilhaam A. Omar, Mazin S. Debe, Khaled Salah, Raja Jayaraman, and Samer Ellahham. "Blockchain for COVID-19: Review, Opportunities, and a Trusted Tracking System." Arabian Journal for Science and Engineering (2020): 117.

[17] Bischoff, P. "COVID-19 App Tracker: Is privacy being sacrificed in a bid to combat the virus?."(2020).

[18] Lucena, Percival, Alécio PD Binotto, Fernanda da Silva Momo, and Henry Kim. "A case study for grain quality assurance tracking based on a Blockchain business network." arXiv preprint arXiv: 1803.07877 (2018).

[19] Srivastava, Gautam, Jorge Crichigno, and Shalini Dhar. "A light and secure healthcare blockchain for iot medical devices." In 2019 IEEE Canadian conference of electrical and computer engineering (CCECE), pp. 1-5. IEEE, 2019.

[20] HASSAN, SHOAIB UL, JINGXIA CHEN, MUHAMMAD AFRASAYAB, ALI AKBAR, and MUHAMMAD AHSAN. "A Systematic Literature Review: Blockchain Based Solutions for IoT's."

[21] Marbouh, Dounia, Tayaba Abbasi, Fatema Maasmi, Ilhaam A. Omar, Mazin S. Debe, Khaled Salah, Raja Jayaraman, and Samer Ellahham. "Blockchain for COVID-19: Review, Opportunities, and a Trusted Tracking System." Arabian Journal for Science and Engineering (2020): 117.

[22] Singh, Rajani, Ashutosh Dhar Dwivedi, and Gautam Srivastava. "Internet of things based blockchain for temperature monitoring and counterfeit pharmaceutical prevention." Sensors 20, no. 14 (2020): 3951.

[23] Aileni, Raluca Maria, and George Suciu. "IoMT: a blockchain perspective." In Decentralised Internet of Things, pp. 199-215. Springer, Cham, 2020.

[24] Bai, Yan, Lingsheng Yao, Tao Wei, Fei Tian, Dong-Yan Jin, Lijuan Chen, and Meiyun Wang."Presumedasymptomatic carrier transmission of COVID-19." Jama 323, no. 14 (2020): 1406-1407.

\section{Authors}

Shoaib ul Hassan got his B.S. degree from Department of Computer Science and Information Technology in University of Sargodha, Sub Campus Bhakkar in Pakistan in 2018 and doing his MS degree from Department of Electronic Information and Artificial Intelligence in Shaanxi University of Science and Technology in China. His research interest is focused on Internet of Things, Android Operating System, Blockchain and Cloud Computing.

Email: shoaibbsit14@gmail.com, ls190312@sust.edu.cn

Jingxia Chen got the B.S. and M.S. degrees from Department of electrical and information engineering in Shaanxi University of Science and Technology in China in 2002 and 2005, respectively and got the Ph.D. degree from School of Computer Science and Engineering, Northwestern Polytechnical University in China in 2020. Now she works as an associate professor in Department of Electronic Information and Artificial Intelligence, Shaanxi University of Science and Technology in China. Her research interest is focus on Machine learning and pattern recognition, EEG signal processing and event detection, and deep learning.

Email: chenjx_sust@foxmail.com

Ali Akbar got his B.S. degree from Department of Computer Science and Information Technology in Iqra University in Pakistan in 2016 and doing his MS degree from Department of Electronic Information and Artificial Intelligence in Shaanxi University of Science and Technology in China. His research interest is focused on Internet of Things, Android Operating System and Cloud Computing.

Email: aa81079@gmail.com

Zaki Ramzan got his B.S. degree from Department of Computer Science in University of Agriculture Faisalabad, Pakistan in 2017 and doing his MS degree from Department of Electronic Information and Artificial Intelligence in Shaanxi University of Science and Technology in China. His research interest is focused on Artificial Intelligence, Internet of Things, Android Operating System and Cloud Computing.

Muhammad Ahsan got his B.S. degree in Electrical Engineering from University of Lahore Pakistan in 2015 and doing his MS degree from Department of Electronic Information and Artificial Intelligence in Shaanxi University of Science and Technology in China. His research interest is focused on Internet of Things and Artificial Intelligence. Email:Engr.ahsanbaba@gmail.com

Muhammad Asim Hafeez got his MSc degree in Gender Studies from Quaid-i-Azam University Islamabad. His Research Interest is focused on currently appearing and over-whelming situations around the Globe. The purpose of research is to explore the facts and figures for Covid-19. Population around the clock and around the globe is effecting badly due to uncontrolled spread of Covid-19. Muhammad Asim Hafeez being social activist \& being companion of different NGO's played his key role in the research topic as mentioned above. He met the attendants of suffering \& took the basic facts which is the vital backbone of the research.

Email: asimhafeez86@gmail.com 